\title{
Structural and electrical properties of ferroelectric $\mathrm{BiFeO}_{3} / \mathrm{HfO}_{2}$ gate stack for nonvolatile memory applications
}

\author{
Nitish Yadav*, Kamal Prakash Pandey ${ }^{*}, \frac{\ddagger}{}$ and Pramod Narayan Tripathi ${ }^{\dagger}$ \\ *Department of Electronics and Communication Engineering, \\ Shambhunath Institute of Engineering and Technology, \\ Allahabad, Uttar Pradesh 211015, India \\ ${ }^{\dagger}$ Department of Electronics and Communication Engineering, \\ Indian Institute of Information Technology, \\ Allahabad, Uttar Pradesh 211015, India \\ †kpandey.ec@gmail.com
}

Received 7 June 2018; Revised 28 September 2018; Accepted 2 October 2018; Published 9 November 2018

\begin{abstract}
Difficulties in the fabrication of direct interface of ferroelectric $\mathrm{BiFeO}_{3}$ on the gate of ferroelectric field effect transistor (FeFET) is well known. This paper reports the optimization and fabrication of ferroelectric/dielectric $\left(\mathrm{BiFeO}_{3} / \mathrm{HfO}_{2}\right)$ gate stack for the FeFET applications. RF magnetron sputtering has been used for the deposition of $\mathrm{BiFeO}_{3}, \mathrm{HfO}_{2}$ films and their stack. X-Ray diffraction (XRD) analysis of $\mathrm{BiFeO}_{3}$ shows the dominant perovskite phase of (104), (110) orientation at $2 \theta=32^{\circ}$ at the annealing temperature of $500^{\circ} \mathrm{C}$. XRD analysis also confirms the amorphous nature of the $\mathrm{HfO}_{2}$ film at annealing temperature of $400^{\circ} \mathrm{C}, 500^{\circ} \mathrm{C}$ and $600^{\circ} \mathrm{C}$. Multiple angle analysis shows the variation ion the refractive index between 2.98-3.0214 for $\mathrm{BiFeO}_{3}$ and $2.74-2.9$ for the $\mathrm{HfO}_{2}$ film with the annealing temperature. Metal/Ferroelectric/Silicon (MFS), Metal/Ferroelectric/Metal (MFM), Metal/Insulator/Silicon (MIS), and Metal/Ferroelectric/Insulator/Silicon (MFIS) structures have been fabricated to obtain the electric characteristic of the ferroelectric, dielectric and their stacks. Electrical characteristics of the MFIS structure show the memory improvement from $2.7 \mathrm{~V}$ for MFS structure to $4.65 \mathrm{~V}$ for MFIS structure with $8 \mathrm{~nm}$ of buffer dielectric layer. This structure also shows the breakdown voltage of $40 \mathrm{~V}$ with data retention capacity greater than $9 \times 10^{9}$ iteration cycles.
\end{abstract}

Keywords: Endurance; ferroelectric; high- $k$ dielectric; memory window; MFIS.

\section{Introduction}

Ferroelectric films have shown its potential in many applications such as transducers, ${ }^{1-4}$ detectors,${ }^{5-7}$ optical devices, ${ }^{8-10}$ dynamic random access memories (DRAMs) ${ }^{11-13}$ and ferroelectric memories. ${ }^{14-16}$ In the last few decades, properties of ferroelectric materials have been widely explored for the memory applications. Ferroelectric materials show spontaneous polarization that can be reversed under the influence of external electric field. ${ }^{17,18}$ Ferroelectric materials have noncentrosymmetric structure, ${ }^{18,19}$ which can be realized by the crystals of double-well potential, where at least one set of ion shows the stable structure by sitting on the same side of the double well giving rise to the region called domain. ${ }^{20,21}$ The ions in the domain sit on the same side of the double wells giving rise to the net dipole moment per unit volume, known as polarization. Net polarization is used to realize two stable states in the memory capacitor that defines the bit "1" or " 0 ". 22,23

Ferroelectric random access memories (FeRAMs) with 1T-1C architecture have been commercially fabricated by different industries which offer the nonvolatile data storage capacity with high reliability and faster read/write speed than the currently existing FLASH memory, ${ }^{24-28}$ but also faces several challenges such as destructive readout and higher cost. $^{24,29,30}$

It is the reason that FeRAM is still not preferred for the large data storage. To simplify the destructive readout and higher cost issue, ferroelectric field effect transistor (FeFET) with direct integration of ferroelectric layer on the MOSFET was proposed. ${ }^{22,31}$ The FeFET structure solves the problem of 1T-1C structure but suffers from ferroelectric-silicon reaction and interdiffusion due to the thermal instability of ferroelectric layer on direct integration with silicon. It is the reason why no FeFET-based FeRAM was commercially fabricated. Further, to solve the issue of ferroelectric and silicon incompatibility, it was proposed that an insulator layer should be introduced between the ferroelectric and silicon resulting into the metal/ferroelectric/insulator/silicon (MFIS) stack at the gate of the MOSFET. ${ }^{32}$ Insulator layer ultimately improves the interface between the silicon and ferroelectric, prevents the $\mathrm{Fe}-\mathrm{Si}$ reaction and interdiffusion, and charge injection from the silicon to ferroelectric layer. ${ }^{33}$

In the present work, $\mathrm{BiFeO}_{3}$ as the ferroelectric and $\mathrm{HfO}_{2}$ as the insulator layer deposited by the RF sputtering have

This is an Open Access article published by World Scientific Publishing Company. It is distributed under the terms of the Creative Commons Attribution 4.0 (CC-BY) License. Further distribution of this work is permitted, provided the original work is properly cited. 
been selected for the MFIS gate stack fabrication. $\mathrm{BiFeO}_{3}$ as a lead-free multiferroic material having high remnant polarization. ${ }^{34-38}$ and $\mathrm{HfO}_{2}$ due to its high bandgap (25) and good thermal stability with silicon ${ }^{39}$ has been selected in this work.

\section{Experimental Procedure}

Silicon substrate $p$-type, $\langle 100\rangle$ orientation having resistivity $1-5 \Omega-\mathrm{cm}$ has been used for the thin film deposition and capacitor fabrications. The substrates have been cleaned using standard Radio Corporation of America (RCA) cleaning procedures prior to the loading of wafers into the vacuum chamber. RF magnetron sputtering operated at the frequency of $13.56 \mathrm{MHz}$ has been used for the thin film deposition of $\mathrm{BiFeO}_{3}(200 \mathrm{~nm})$ and $\mathrm{HfO}_{2}$. The system has been pumped down to $5 \times 10^{-6}$ mbar and the deposition was carried out at $9 \times 10^{-3}$ mbar chamber pressure. Deposited film annealing has been carried out in a quartz tube furnace in the presence of nitrogen for different temperature range. Top platinum and aluminum metal contacts have been fabricated through a shadow mask of circular opening $3.14 \times 10^{-4} \mathrm{~cm}^{2}$ using sputtering and thermal evaporation processes, respectively. For the Metal/Ferroelectric/Metal (MFM) structure, $100 \mathrm{~nm}$ top and bottom platinum electrode has been fabricated by DC sputtering at the power of $100 \mathrm{~W}$. X-Ray diffraction (XRD) analysis for the crystal orientation detection and multiple angle ellipsometric analysis for the film thickness, refractive index and absorption constant detection has been used.
Metal/Ferroelectric/Silicon (MFS), MFM, Metal/Insulator/ Silicon (MIS), and Metal/Ferroelectric/Insulator/Silicon (MFIS) structures have been fabricated and electrically characterized to obtain the capacitance, leakage current density, remnant charges, coercive electric field, breakdown voltage and endurance using keithley 4200 Semiconductor characterization system.

\section{Results and Discussion}

Figures 1(a) and 1(b) show the XRD pattern of the $\mathrm{BiFeO}_{3}$ and $\mathrm{HfO}_{2}$ film annealed at different temperature, respectively.

$\mathrm{BiFeO}_{3}$ film annealed at $500^{\circ} \mathrm{C}$ shows the maximum intensity peak at $2 \theta=32^{\circ}$ indicating the (104), (110) orientation and perovskite phase at the annealing temperature of $500^{\circ} \mathrm{C}$. Several secondary intensity peaks have been observed at the annealing temperatures of $400^{\circ} \mathrm{C}$ and $600^{\circ} \mathrm{C}$, which show the existence of different phases possible due to the noncrystallized deposited film at $400^{\circ} \mathrm{C}$ and due to the loss of film stoichiometry at $600^{\circ} \mathrm{C}$. Amorphous $\mathrm{HfO}_{2}$ film structure has been evident from the XRD pattern, where no significant intensity has been observed. Ellipsometric analysis has been carried out to obtain the refractive index at different annealing temperature and the results obtained are shown in Fig. 2.

Maximum refractive indices of 3.03 and 2.9 have been observed for the $\mathrm{BiFeO}_{3}$ and $\mathrm{HfO}_{2}$ film at the annealing temperature of $500^{\circ} \mathrm{C}$. Higher refractive index indicates the

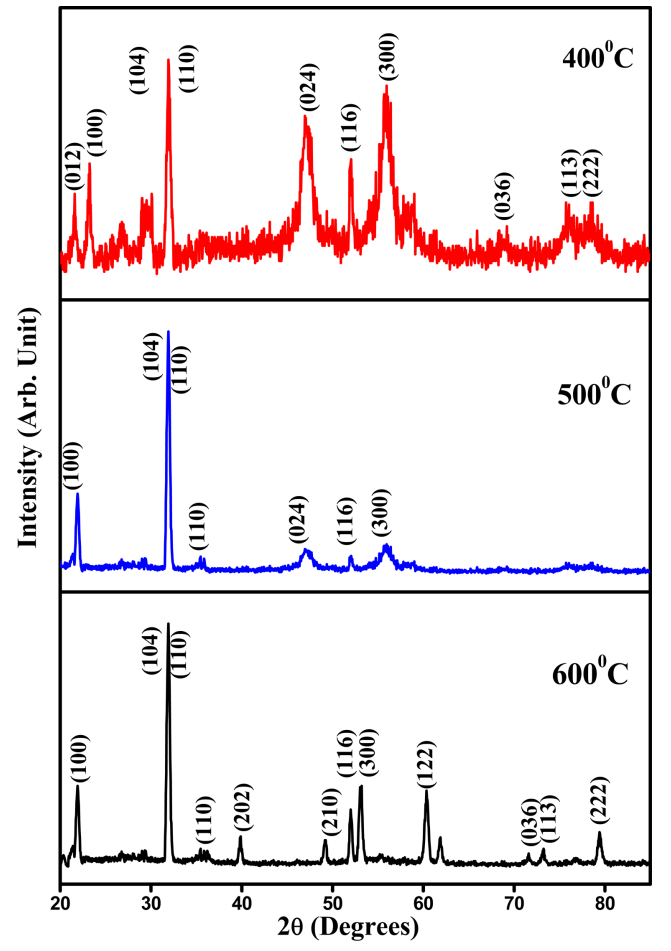

(a)

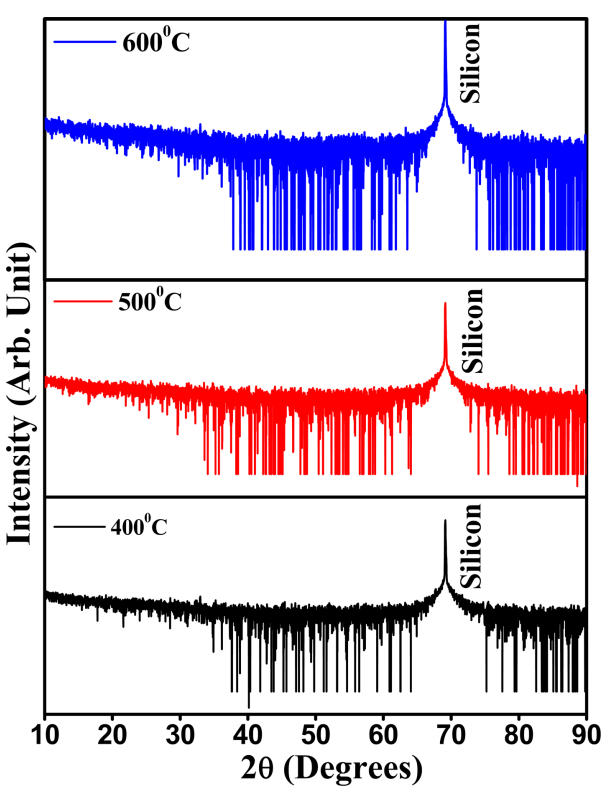

(b)

Fig. 1. XRD pattern of (a) $\mathrm{BiFeO}_{3}$, and (b) $\mathrm{HfO}_{2}$ film annealed at $400^{\circ} \mathrm{C}, 500^{\circ} \mathrm{C}$ and $600^{\circ} \mathrm{C}$. 


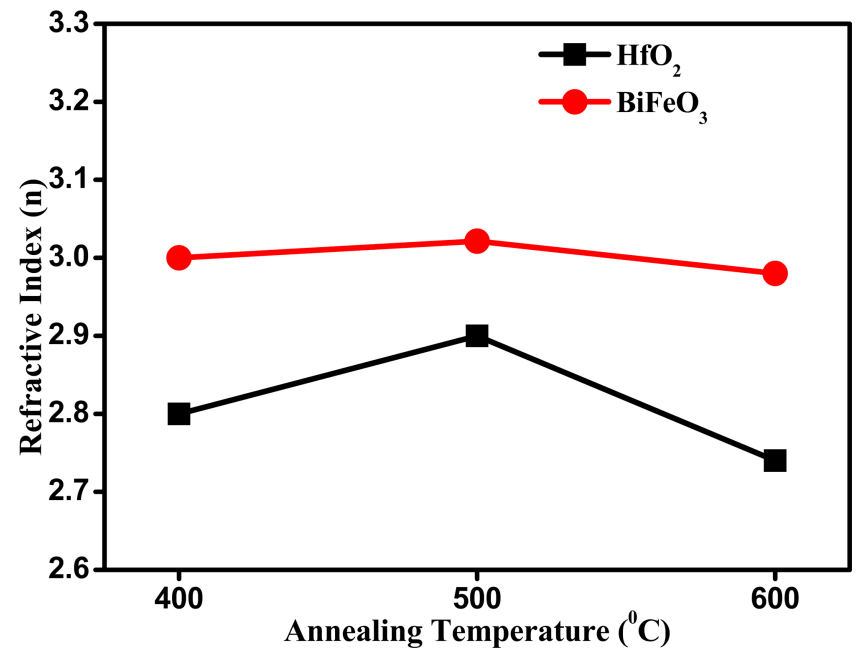

Fig. 2. Refractive index of $\mathrm{BiFeO}_{3}$ and $\mathrm{HfO}_{2}$ film annealed at $400^{\circ} \mathrm{C}, 500^{\circ} \mathrm{C}$ and $600^{\circ} \mathrm{C}$.

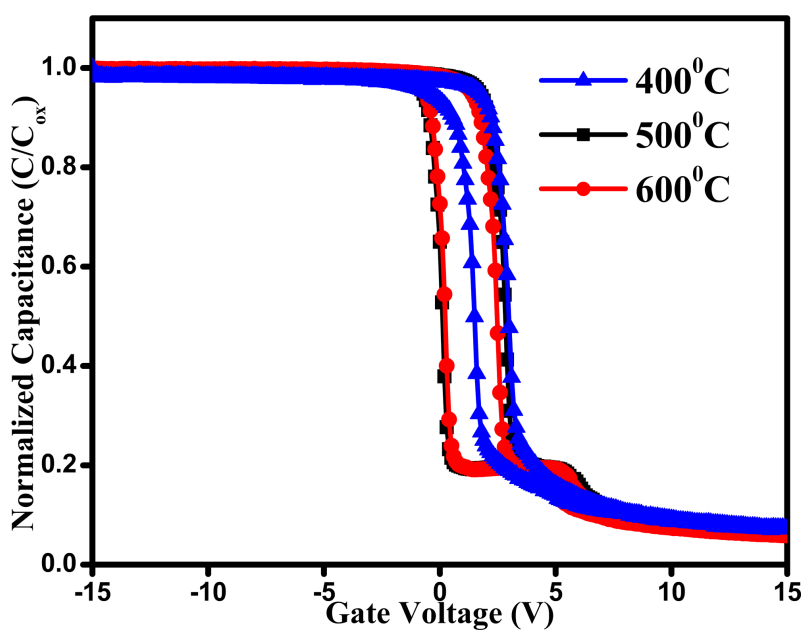

(a) increased film density, ${ }^{40,41}$ possibly due to the film crystallization, as evident from the XRD data in case of the $\mathrm{BiFeO}_{3}$ film. After the structural characterization, MFS structure has been fabricated and electrically characterized to obtain the $C-V$ and $J-V$ parameters shown in Fig. 3 .

Maximum memory window of $2.7 \mathrm{~V}$ has been observed for the MFS structure with ferroelectric film annealed at $500^{\circ} \mathrm{C}$. Maximum memory window at $500^{\circ} \mathrm{C}$ is due to the crystallized film even evident from the XRD and ellipsometric analysis. The memory window improved from $1.5 \mathrm{~V}$ to $2.7 \mathrm{~V}$ with the annealing temperature from $400^{\circ} \mathrm{C}$ to $500^{\circ} \mathrm{C}$ and further reduced to $2.3 \mathrm{~V}$ as the annealing temperature increased to $600^{\circ} \mathrm{C}$. The leakage current density of the orders $10^{-5} \mathrm{~A} / \mathrm{cm}^{2}$ and $10^{-6} \mathrm{~A} / \mathrm{cm}^{2}$ has been observed for films annealed at different temperature at the voltage bias of $-10 \mathrm{~V}$ and $+10 \mathrm{~V}$, respectively. As the ferroelectric film annealed at $500^{\circ} \mathrm{C}$ shows crystalline perovskite phase and maximum memory window, we have fabricated the MFM structure to

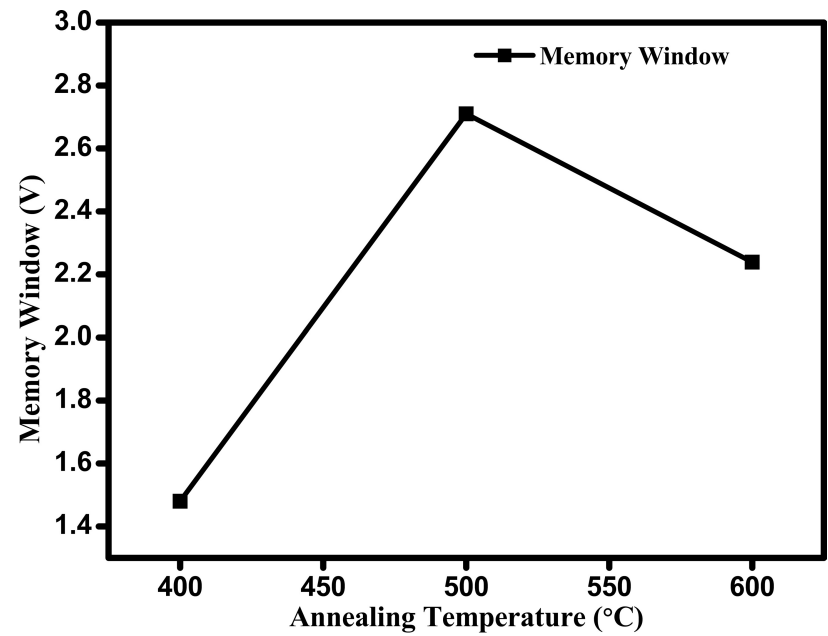

(b)

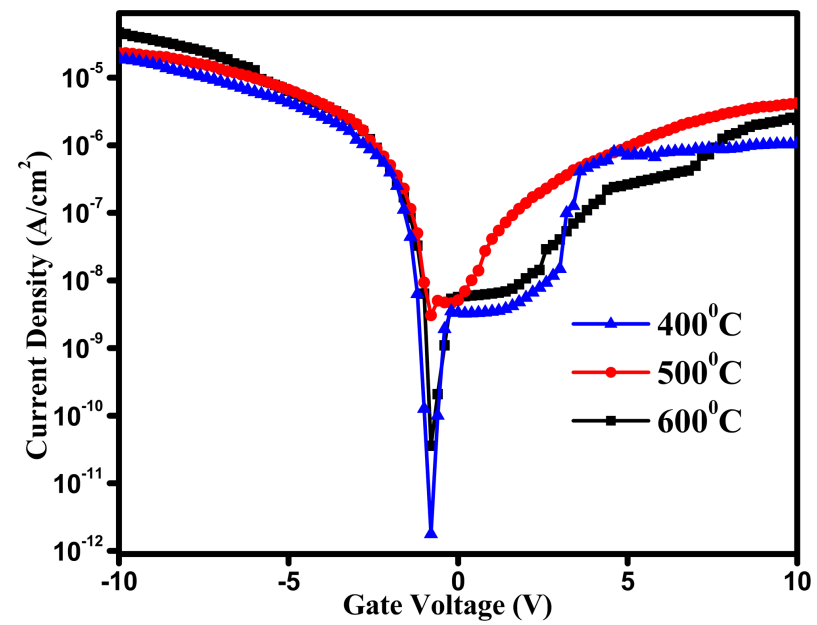

(c)

Fig. 3. (a) $C-V$, (b) memory window versus annealing temperature, and (c) $J-V$ characteristics of the MFS structures annealed at $400^{\circ} \mathrm{C}$, $500^{\circ} \mathrm{C}$ and $600^{\circ} \mathrm{C}$. 


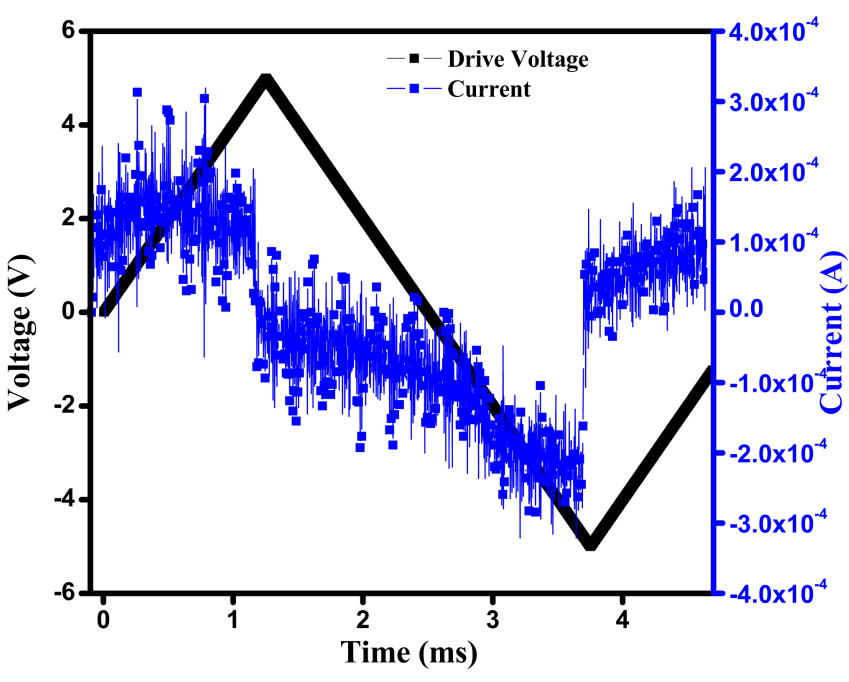

(a)

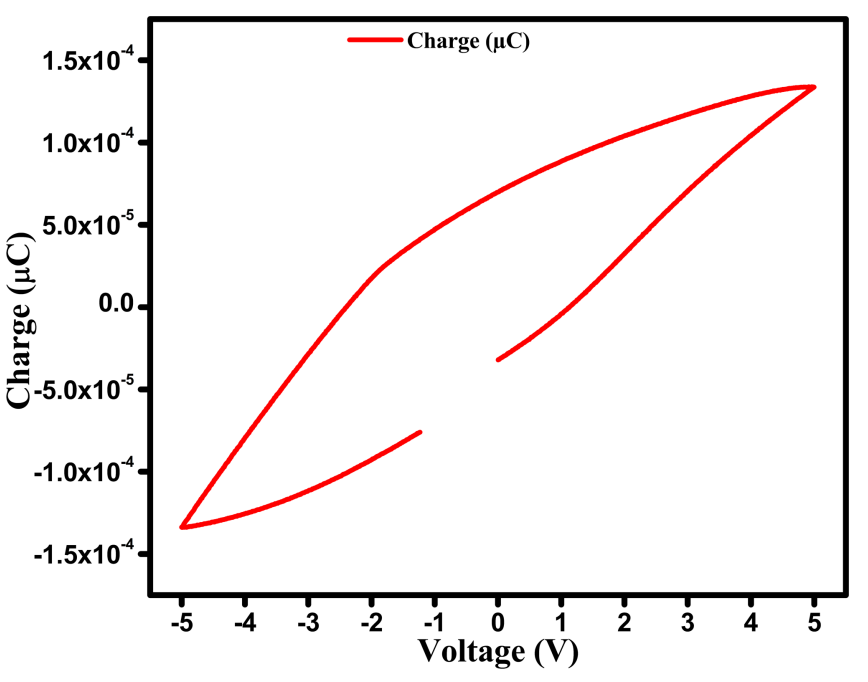

(b)

Fig. 4. (a) Sample input voltage and current, (b) charge versus voltage hysteresis loop for the MFM structure with ferroelectric film annealed at $500^{\circ} \mathrm{C}$.

obtain the hysteresis characteristic with ferroelectric film annealed at $500^{\circ} \mathrm{C}$, as shown in Fig. 4 .

The MFM structure shows the proper hysteresis loop with remanent charge $7.07 \times 10^{-5} \mu \mathrm{C}$ and coercive voltage of $-2.4 \mathrm{~V}$ for the input voltage sweep of $\pm 5 \mathrm{~V}$. After the electrical characterization of ferroelectric film, the electric properties of dielectric film have been investigated by obtaining the $C-V$ and $J-V$ characteristics of the MIS structure, as shown in Fig. 5.

Electrical characteristics show the proper interface of $\mathrm{HfO}_{2}$ film with silicon with low interface defect density as observed from the $C-V$ characteristics. Leakage current density of the orders $10^{-1} \mathrm{~A} / \mathrm{cm}^{2}$ and $10^{-6} \mathrm{~A} / \mathrm{cm}^{2}$ has been observed for the gate voltage of $-10 \mathrm{~V}$ and $+10 \mathrm{~V}$, respectively. Higher leakage current density at the negative gate bias is due to the charge injection from the silicon to the insulator layer.

After ferroelectric and insulator film optimization and electrical characterization, the MFIS gate stack has been fabricated with insulator layer of thicknesses 3, 8 and $13 \mathrm{~nm}$. The MFIS structures were electrically characterized to obtain the capacitance versus voltage characteristics, as shown in Fig. 6.

Maximum memory window of $4.65 \mathrm{~V}$ has been observed for the insulator layer of $8 \mathrm{~nm}$ thickness at the voltage sweep of $\pm 15 \mathrm{~V}$. Lower memory window of $1.7 \mathrm{~V}$ and $3.1 \mathrm{~V}$ has been observed for the insulator layer thicknesses of $3 \mathrm{~nm}$ and $13 \mathrm{~nm}$, respectively. For $3 \mathrm{~nm}$ buffer layer, lower memory

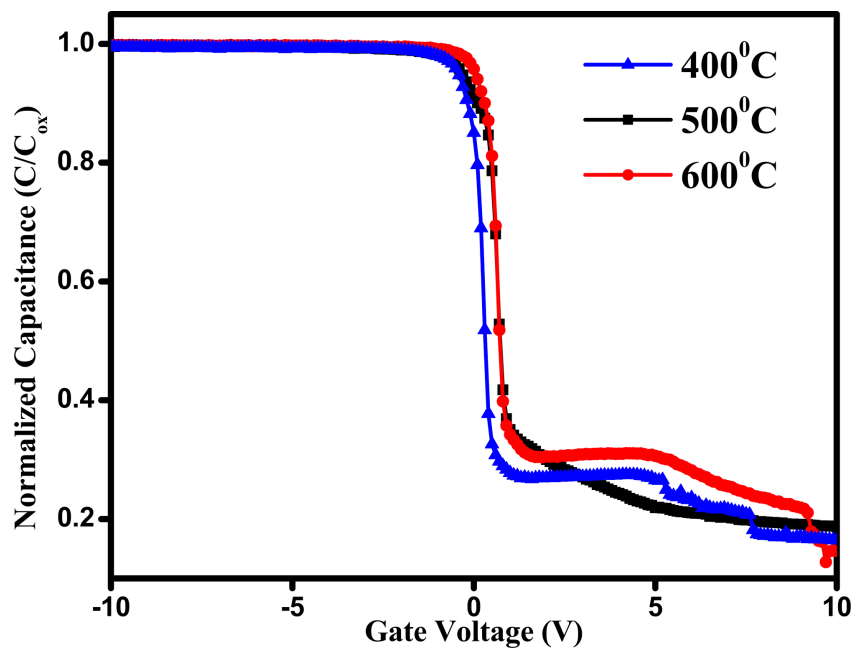

(a)

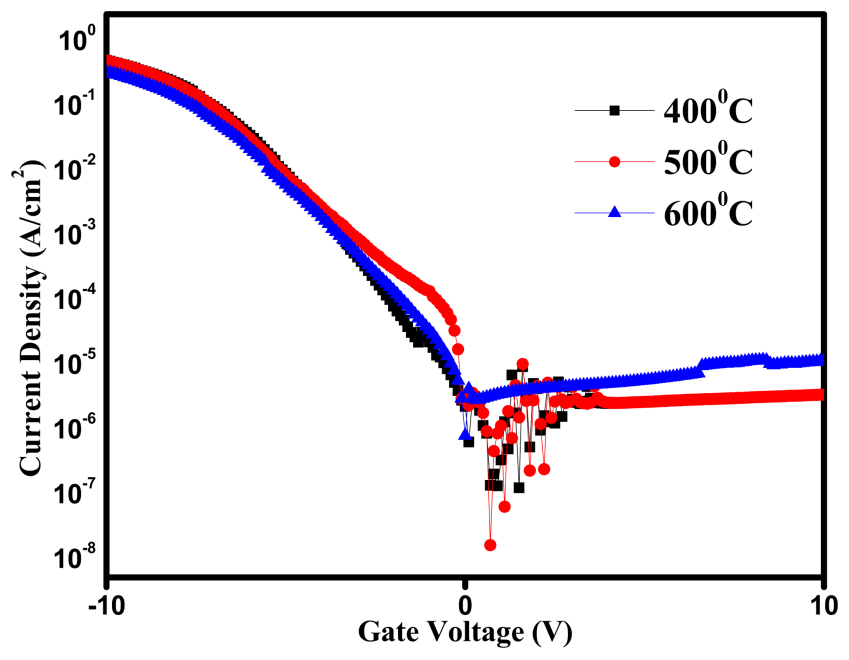

(b)

Fig. 5. (a) $C-V$ and (b) $J-V$ characteristics of the MIS structure with insulator film annealed at $400^{\circ} \mathrm{C}, 500^{\circ} \mathrm{C}$ and $600^{\circ} \mathrm{C}$. 


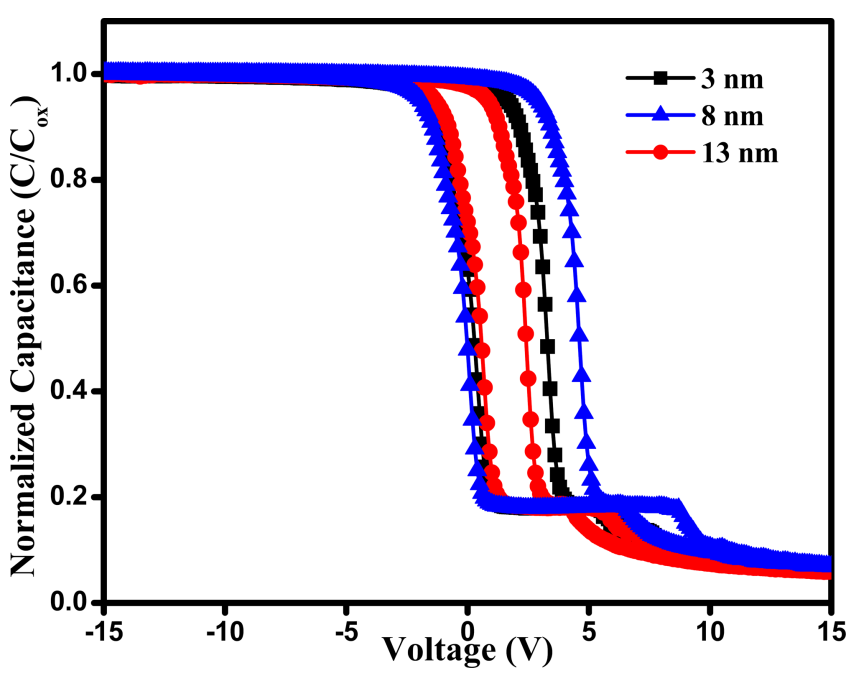

(a)

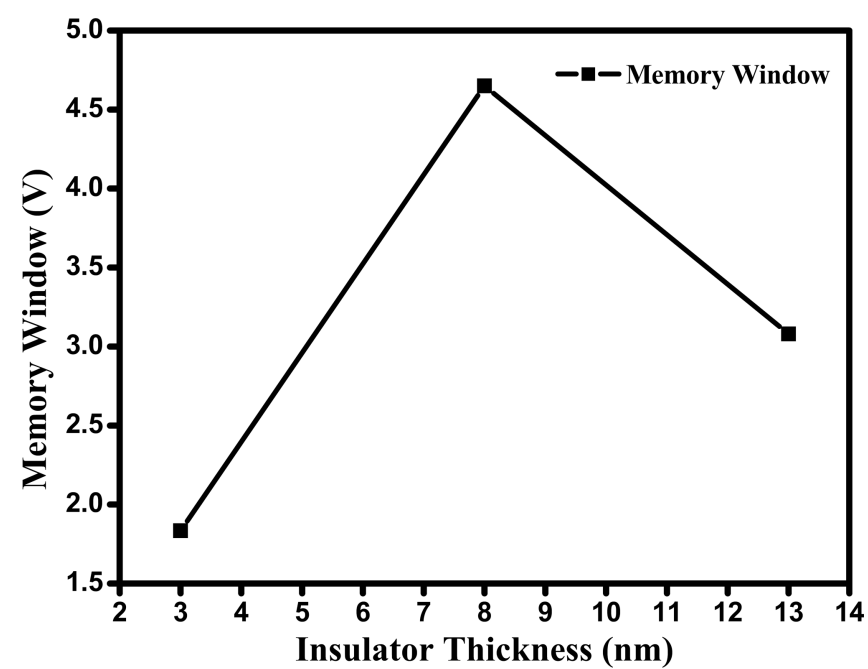

(b)

Fig. 6. (a) $C-V$, and (b) memory window characteristic for different insulator layer thickness.

window is possible due to the diffusion of $\mathrm{BiFeO}_{3}$ through the buffer layer to the silicon substrate providing the direct contact path for the charges to flow, hence increased leakage and reduced memory window. Buffer layer of lower thickness does not provide sufficient shielding to the ferroelectric film to diffuse through it and reach to the silicon substrate. Also, the charges at high electric film tunnel through the buffer layer of low thickness resulting in the increased leakage current, hence low memory window and data retention characteristics. For $13 \mathrm{~nm}$ buffer layer, the buffer layer thickness is increased which improves the charge leakage and ferroelectric diffusion to silicon but have the disadvantage of higher voltage drop across it. Due to higher voltage drop, the net voltage available to the ferroelectric layer decreased

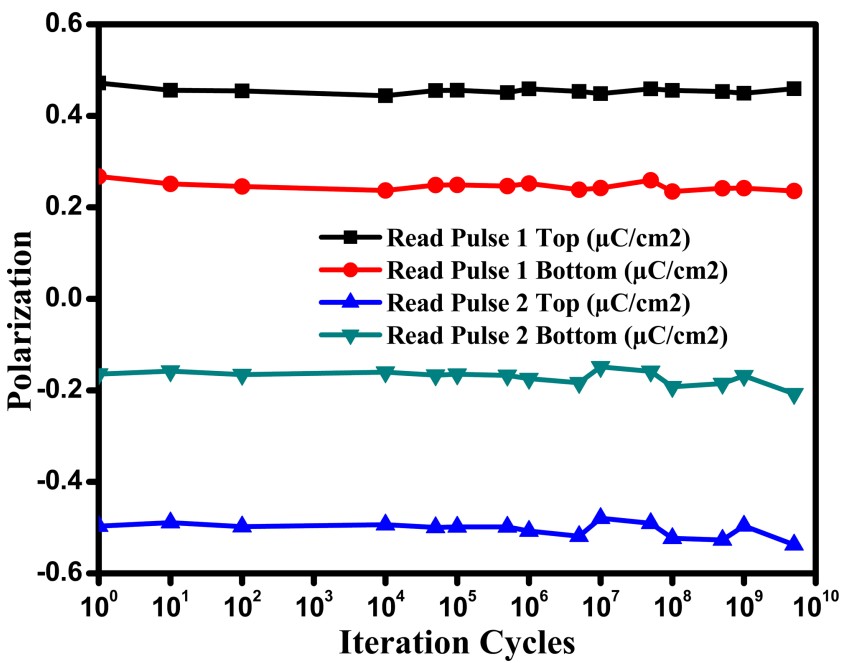

Fig. 7. Data retention characteristic of the MFIS structure with $8 \mathrm{~nm}$ buffer layer annealed at $500^{\circ} \mathrm{C}$. hence lower polarization and lower memory window. The $8 \mathrm{~nm}$ buffer layer thickness provides the sufficient potential barrier to the charge tunneling from ferroelectric to silicon (less than $13 \mathrm{~nm} \mathrm{HfO}_{2}$ film) along with the ferroelectric diffusion to silicon. At the same time, the voltage drop across the buffer layer is low as compared to the $13 \mathrm{~nm} \mathrm{HfO}_{2}$ film. These are the reasons which improve the memory window of the MFIS structure with $8 \mathrm{~nm}$ buffer layer as compared to the $3 \mathrm{~nm}$ and $13 \mathrm{~nm}$ buffer layers. Now, the MFIS structure with $8 \mathrm{~nm}$ buffer layer has been characterized to investigate the retention time and the result obtained is shown in Fig. 7.

Results clearly indicate that the MFIS structure shows no decrease in the positive and negative polarizations for the iteration cycles up to $9 \times 10^{9}$. The breakdown characteristic

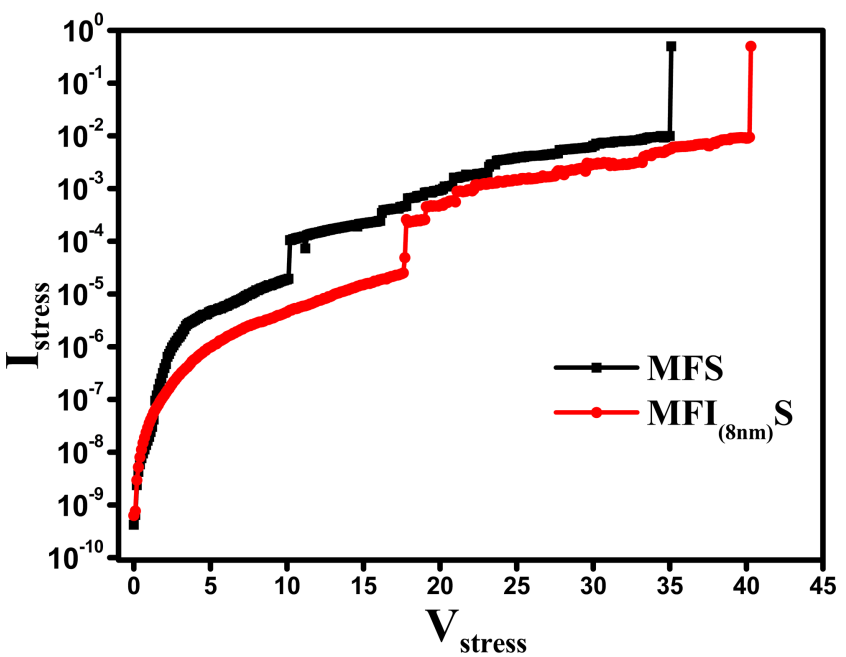

Fig. 8. Breakdown voltage of MFS and $\mathrm{MFI}_{8 \mathrm{~nm}} \mathrm{~S}$ structure with ferroelectric and dielectric films annealed at $500^{\circ} \mathrm{C}$. 
of the same structure and the MFS structure annealed at $500^{\circ} \mathrm{C}$ has been investigated and the result obtained is shown in Fig. 8.

With the introduction of the $8 \mathrm{~nm}$ buffer layer, the breakdown voltage of the MFIS structure improved to $41 \mathrm{~V}$ from $35 \mathrm{~V}$ of the MFS structure. Improved breakdown voltage is due to the increased stack thickness and the strength provided by the $\mathrm{HfO}_{2}$ layer along with ferroelectric layer.

\section{Conclusion}

In conclusion, perovskite $\mathrm{BiFeO}_{3}$ film has been prepared at the annealing temperature of $500^{\circ} \mathrm{C}$. Effect of buffer layer sandwiched between ferroelectric and silicon substrate improves the memory window, breakdown and data retention capacity. The fabricated structure can be used as a potential candidate for the FeFET nonvolatile memory application.

\section{References}

${ }^{1}$ S. E. Park and T. R. Shrout, Characteristics of relaxor-based piezoelectric single crystals for ultrasonic transducers, IEEE Trans. Ultrason. Ferroelectr. Freq. Control 44, 1140 (1997).

${ }^{2}$ J. J. Bernstein et al., Micromachined high frequency ferroelectric sonar transducers, IEEE Trans. Ultrason. Ferroelectr. Freq. Control 44, 960 (1997).

${ }^{3}$ D. Damjanovic, Materials for high temperature piezoelectric transducers, Curr. Opin. Solid State Mater. Sci. 3, 469 (1998).

${ }^{4}$ J. M. Herbert, Ferroelectric Transducers and Sensors (Gordon and Breach Science Publishers, New York, 1982).

${ }^{5}$ H. Lin, N. J. Wu, F. Geiger, K. Xie and A. Ignatiev, A ferroelectric-superconducting photodetector, J. Appl. Phys. 80, 7130 (1996).

${ }^{6} \mathrm{R}$. Watton, Ferroelectric materials and devices in infrared detection and imaging, Ferroelectrics 91, 87 (1989).

${ }^{7}$ A. M. Glass, Ferroelectric Sr1- $x \mathrm{Ba} x \mathrm{Nb} 2 \mathrm{O} 6$ as a fast and sensitive detector of infrared radiation, Appl. Phys. Lett. 13, 147 (1968).

${ }^{8} \mathrm{~S}$. Y. Yang et al., Above-bandgap voltages from ferroelectric photovoltaic devices, Nat. Nanotechnol. 5, 143 (2010).

${ }^{9} \mathrm{~S}$. H. Wemple and M. DiDomenico, Oxygen-octahedra ferroelectrics. II. Electro-optical and nonlinear-optical device applications, J. Appl. Phys. 40, 735 (1969).

${ }^{10}$ U. Kenji, Ferroelectric Devices (CRC Press, New York, 2000).

${ }^{11}$ O. Auciello, J. F. Scott and R. Ramesh, The physics of ferroelectric memories, Phys. Today 51, 22 (1998).

${ }^{12}$ J. F. Scott, High-dielectric constant thin films for dynamic random access memories (DRAM), Annu. Rev. Mater. Sci. 28, 79 (1998).

${ }^{13}$ A. I. Kingon, S. K. Streiffer, C. Basceri and S. R. Summerfelt, High-permittivity perovskite thin films for dynamic randomaccess memories, MRS Bull. 21, 46 (1996).

${ }^{14}$ A. Carlos et al., Ferroelectric memories, Ferroelectrics 104, 241 (1990).

${ }^{15}$ J. F. Scott et al., Ferroelectric memories, Science 246, 1400 (1989).
${ }^{16}$ J. F. Scott, Ferroelectric memories today, Ferroelectrics 236, 247 (2000).

${ }^{17}$ S. C. Abrahams, S. K. Kurtz and P. B. Jamieson, Atomic displacement relationship to curie temperature and spontaneous polarization in displacive ferroelectrics, Phys. Rev. 172, 551 (1968).

${ }^{18}$ K. M. Ok, E. O. Chi and P. S. Halasyamani, Bulk characterization methods for non-centrosymmetric materials: Second-harmonic generation, piezoelectricity, pyroelectricity, and ferroelectricity, Chem. Soc. Rev. 35, 710 (2006).

${ }^{19}$ R. Ramesh and N. A. Spaldin, Multiferroics: Progress and prospects in thin films, Nat. Mater. 6, 21 (2007).

${ }^{20}$ L. D. Landau, On the theory of phase transitions, Zh. Eks. Teor. Fiz. 7, 19 (1937).

${ }^{21}$ A. F. Devonshire, Theory of barium titanate, Dublin Philos. Mag. J. Sci. 40, 1040 (1949).

${ }^{22}$ G. C. Messenger, Ferroelectric Memories (Defense Nuclear Agency, Washington, DC, 1988).

${ }^{23}$ K. Rabe, C. Ahn and J. M. Triscone, Physics of Ferroelectronics: A Modern Perspective (Springer, Verlag, Berlin, Heidelberg, 2007).

${ }^{24}$ M. Dawber, K. M. Rabe and J. F. Scott, Physics of thin-film ferroelectric oxides, Rev. Mod. Phys. 77, 1083 (2005).

${ }^{25}$ S. O. Park, B. J. Bae, D. C. Yoo and U.-I. Chung, Ferroelectric random access memory, Nanotechnology 3, 397 (2010).

${ }^{26}$ Ramtron Announces 1T/1C 256Kb Serial FRAM Data Collection Subsystem; Newest FRAM Problem Solver Product Targets Digital Metering Applications. Free Online Library. Available at: https://www.thefreelibrary.com/Ramtron+Announces+1T\%2F1C $+256 \mathrm{~Kb}+$ Serial+FRAM+Data+Collection+Subsystem\%3B ...a083277132. Accessed on 20th May 2018.

${ }^{27}$ Fujitsu Successfully Develops 1T1C FRAM Memory Cell Technology. Available at: https://pr.fujitsu.com/en/news/2000/03/13. html. Accessed on 20th May 2018.

${ }^{28}$ SAMSUNG's FRAM has an advanced (and simplified) cell structure with one transistor and one capacitor (1T/1C). In addition, double-layer metal circuit processing has been applied to enhance integration SAMSUNG. Available at: http://www. samsung.com/us/news/newsPreviewRead.do?news_seq $=247$. Accessed on 20th May 2018.

${ }^{29} \mathrm{~K}$. Kim, Technology perspective for 1T/1C fram, Integr. Ferroelectr. 25, 149 (1999).

${ }^{30}$ R. Bez and A. Pirovano, Non-volatile memory technologies: Emerging concepts and new materials, Mater. Sci. Semicond. Process. 7, 349 (2004).

${ }^{31}$ Y. Tarui, T. Hirai, K. Teramoto, H. Koike and K. Nagashima, Application of the ferroelectric materials to ULSI memories, Appl. Surf. Sci. 114, 656 (1997).

${ }^{32}$ B.-E. Park et al. (eds.), Ferroelectric-gate field effect transistor memories, Topics in Applied Physics, Vol. 131 (Springer-Verlag, Berlin, 2016), pp. 3-20, doi:10.1007/978-94-024-0841-6_1.

${ }^{33} \mathrm{Y}$. Arimoto and H. Ishiwara, Current status of ferroelectric random-access memory, MRS Bull. 29, 823 (2004).

${ }^{34}$ K. Y. Yun, D. Ricinschi, T. Kanashima, M. Noda and M. Okuyama, Giant ferroelectric polarization beyond $150 \mu \mathrm{C} / \mathrm{cm}^{2}$ in $\mathrm{BiFeO}_{3}$ thin film, Jpn. J. Appl. Phys. Lett. 43, L647 (2004).

${ }^{35} \mathrm{~J}$. Wang et al., Epitaxial $\mathrm{BiFeO}_{3}$ multiferroic thin film heterostructures, Science 299, 1719 (2003). 
${ }^{36}$ S. Goswami, D. Bhattacharya, P. Choudhury, B. Ouladdiaf and T. Chatterji, Multiferroic coupling in nanoscale $\mathrm{BiFeO}_{3}$, Appl. Phys. Lett. 99, 073106 (2011).

${ }^{37}$ I. Sosnowska, T. P. Neumaier and E. Steichele, Spiral magnetic ordering in bismuth ferrite, J. Phys. C, Solid State Phys. 15, 4835 (1982).

${ }^{38}$ T. Zhao et al., Electrical control of antiferromagnetic domains in multiferroic $\mathrm{BiFeO}_{3}$ films at room temperature, Nat. Mater. 5, 823 (2006).
${ }^{39}$ J. Robertson, High dielectric constant oxides, Eur. Phys. J. Appl. Phys. 28, 265 (2004).

${ }^{40}$ K. K. Shih and D. B. Dove, Deposition of aluminum oxide films with high refractive index, J. Vac. Sci. Technol. A, Vac. Surf. Films 12, 321 (1994).

${ }^{41}$ M. H. Suhail, G. M. Rao and S. Mohan, Dc reactive magnetron sputtering of titanium-structural and optical characterization of $\mathrm{TiO}_{2}$ films, J. Appl. Phys. 71, 1421 (1992). 